\title{
Effects of 5-aminolevulinic Acid on the Photosynthesis, Antioxidant System, and $\alpha$-Bisabolol Content of Matricaria recutita
}

\author{
Xiaomeng $\mathrm{LIU}^{1}$, $\mathrm{Li} \mathrm{ZHU}^{1}$, Qiling SONG ${ }^{1}$, Jie $\mathrm{CHANG}^{2,3}$, Jiabao $\mathrm{YE}^{1}$, \\ Weiwei $\mathrm{ZHANG}^{1}$, Yongling $\mathrm{LIAO}^{1}$, Feng XU ${ }^{1 *}$

\begin{abstract}
${ }^{1}$ Yangtze University, College of Horticulture and Gardening, Jingzhou 434025, Hubei, China; LiuXM925@163.com; zhuli322@163.com; song70@126.com;yejiabao13@163.com;wwzhangchn@163.com; liaoyongling@yeah.net;xufeng198@126.com (*correspondingauthor)

${ }^{2}$ Hubei Collaborative Innovation Center of Targeted Antitumor Drug, Jingmen 448000, Hubei, China; $18986662379 @ 163 . c 0 m$

3 Jingchu University of Technology, College of Chemical Engineering and Pharmacy, Jingmen, 448000, Hubei, China
\end{abstract}

\begin{abstract}
Matricaria recutita is a widely used medicinal plant with broad pharmacological effects, and $\alpha$-bisabolol is the main active ingredient of this plant. To improve its $\alpha$-bisabolol content, $M$. recutita was sprayed with different concentrations (1.0, 2.0, and $4.0 \mathrm{mmol} \cdot \mathrm{L}^{-1}$ ) of 5 -aminolevulinic acid (ALA) or with water as a control to study the effects of ALA treatment on the photosynthesis, antioxidant system, and $\alpha$-bisabolol content of $M$. recutita. Results showed that the photosynthetic rate, transpiration rate, stomatal conductance, intercellular $\mathrm{CO}_{2}$ concentration, soluble protein, total amino acids, soluble sugar, and $\alpha$-bisabolol of $M$. recutita were significantly increased. Moreover, the activities of superoxide dismutase, peroxidase, and catalase of $M$. recutita were also enhanced by ALA treatment. Optimal results were obtained when the concentration of ALA was $2.0 \mathrm{mmol} \cdot \mathrm{L}^{-1}$. Results showed that ALA treatment could improve the $\alpha$-bisabolol content of $M$. recutita, and the underlying physiological mechanism was analyzed. ALA treatment was an effective measure for improving the medicinal value of $M$. recutita.
\end{abstract}

Keywords: antioxidant system; $\alpha$-bisabolol; 5-aminolevulinic acid; Matricaria recutita; photosynthesis

\section{Introduction}

Chamomile (Matricaria recutita L.) is an annual or perennial herb of Chrysanthemum, which is native to Europe (Farhoudi et al., 2013). The whole plant of $M$. recutita is aromatic and rich in essential oil, and this plant exhibits various effects such as anti-inflammatory, antioxidant, antibacterial, anti-inflammatory, spasm relief, and analgesic (Srivastava et al., 2010). Thus, this medicinal plant is remarkably developed and has high utilization value. $M$. recutita is widely used as additive to spices, cosmetics, medicines, and other products (Ma et al., 2007). The main active ingredients of the essential oil of $M$. recutita are terpene compounds, of which $\alpha$-bisabolol is the most important terpenoid (Raal et al., 2012). Therefore, improving $\alpha$-bisabolol content in $M$. recutita has become an important concern in the cultivation of this plant.

5-Aminolevulinic acid (ALA) is a non-protein amino acid widely found in the living cells of bacteria, fungi, animals, and plants (Xu et al., 2015). ALA is a key precursor of many tetrapyrrols, such as including porphyrins for chlorophyll and haeme biosynthesis (Naeem et al., 2011). Moreover, ALA has great potential in agriculture as a new type of plant growth regulator (Watanabe et al., 2000). High dosage of ALA has been used as a biodegradable photodynamic herbicide in agricultural production (Duke et al., 1994). Meanwhile, low concentration of exogenous ALA has many physiological effects on plant growth regulation, such as the enhancement of yields of some plants (Hotta et al., 1997a). Hotta et al. (1997b) showed that low concentrations of ALA can improve plant photosynthesis and increase plant growth. Moreover, numerous studies have shown that low ALA concentration can promote salt resistance in cotton seedlings (Liu et al., 2011), cold tolerance in rice (Hotta et al., 1998), and herbicide tolerance in oilseed (Zhang et al., 2008). Under low light and cold environment, ALA can also improve chlorophyll content, gas exchange ability, and photosynthesis rate $\left(P_{\mathrm{n}}\right)$ of melon seedling leaf (Wang et al., 2004). Low concentration of ALA could accelerate the production of reactive oxygen species, such as $\mathrm{H}_{2} \mathrm{O}_{2}$, and increase the 
activity of key antioxidant enzymes, such as superoxide dismutase (SOD), catalase (CAT), ascorbate peroxidase (APX), and dehydroascorbate reductase (DHAR) (Xu et al., 2009). In addition, our previous work also showed that exogenous ALA could increase the biosynthesis of anthocyanin in peach skin (Ye et al., 2017). Therefore, there is reason to believe that ALA can promote plant secondary metabolites, such as the synthesis of flavonoids and terpenoid compounds. However, the application of ALA in $M$. recutita has not been reported yet. In this study, the effects of ALA on the growth, photosynthesis, antioxidant system, and $\alpha$-bisabolol content of $M$. recutita were investigated by spraying $M$. recutita with different concentrations of exogenous ALA solutions. Theoretical and experimental bases for improving the medicinal value of $M$. recutita were provided.

\section{Materials and Methods}

\section{Plant materials and treatments}

The experiment was conducted in the glass greenhouse of Yangtze University from November 2014 to May 2015. The test material is the seed of $M$. recutita sown in a nutrition bowl $(20 \times 20 \mathrm{~cm})$ after immersion and germination on November 6,2014 . The culture medium was vermiculite, coconut shell powder and pearl, uniformly mixed with the ratio of 1: 1: 1 and placed in the greenhouse for seedling cultivation. The day/night light cycle was 16 $\mathrm{h} / 8 \mathrm{~h}$, temperature was $24^{\circ} \mathrm{C} / 18^{\circ} \mathrm{C}$, and relative humidity was $70 \%$. Two weeks after seed germination, $M$. recutita was planted in a nutrition bowl, and three strains of $M$. recutita were planted in each pot, for a total of 50 pots.

Then, 15 days after transplantation, 36 pots of morphologically uniform $M$. recutita were selected and foliary sprayed with aqueous solution of ALA at 1.0, 2.0, and $4.0 \mathrm{mmol}^{-1}$. In the control treatment, distilled water was used instead of ALA. Each treatment was repeated thrice, and each replicate involved three pots. ALA is easily decomposed under irradiation. Thus, foliar spraying with a handheld atomizer was performed at night when the light was weak. The plants were treated once every two weeks, for a total of 8 sprays. During this period, the cultivation and management measures were undertaken to maintain consistency until $M$. recutita blossomed. Then, the whole plants were marked, frozen (roots were cleaned, and surface water was drained with filter paper), and stored in liquid nitrogen for subsequent determination of fresh weight and physiological characteristics. Three technical replicates were used.

\section{Determination of growth and physiology characteristics}

Fresh weight and dry weight (ground and underground), which were expressed in milligrams, were measured separately after $M$. recutita blossom. After sample collection, the roots were cleaned, and moisture on the surface was drained with a filter paper. The samples were immediately weighed and then placed in a hot-air oven at $80^{\circ} \mathrm{C}$ until weighed at constant weight.

Photosynthetic parameters, namely, intercellular $\mathrm{CO}_{2}$ concentration (Ci), stomatal conductance $(G s)$, photosynthetic rate $(P n)$, and transpiration rate $(T r)$, were measured using the Li6400 portable photosynthesis system on the fifth leaf of each M. recutita from top to bottom. Given that the leaf area of M. recutita was less than $6 \mathrm{~cm}^{2}$, a leaf area meter should be used to measure the leaf area of the collected fresh leaves.

Chlorophyll $a$, chlorophyll $b$, and total chlorophyll contents were determined according to the method of Wellburn (1994). Chlorophyll content [mg/g FW (fresh weight)] was calculated at 665 and $649 \mathrm{~nm}$ by spectrophotometer (UV-2800, Unico, GER).

Soluble sugar content was determined using anthrone colorimetric method (Moustakas et al., 2011). Fresh tissue samples (5 g) were collected and ground into a homogeneous slurry. Then, the ground samples were extracted with $6 \mathrm{ml}$ of sulfosalicylic acid for $2 \mathrm{~h}$, filtered by a funnel, and centrifuged at $4,000 \times g$ for $5 \mathrm{~min}$. The supernatant after centrifugation was used to calculate the soluble sugar content $[\mathrm{mg} / \mathrm{g} \mathrm{FW}$ (fresh weight)] at a wavelength of $630 \mathrm{~nm}$ by a spectrophotometer.

Soluble protein contents [mg/g FW (fresh weight)] were determined according to the specification of a protein quantification kit.

The activities of antioxidant enzymes, namely SOD, CAT, POD, APX, were determined according to the instructions of corresponding kits.

$\alpha$-Bisabolol in of $M$. recutita was determined according to the method of Irmisch et al. (2012). Essential oil from the sample of the test tissue was extracted and analyzed by gas chromatography-mass spectrometry (GC-MS, Agilent $6890 \mathrm{~N}$ gas chromatography system and Agilent 5975B mass spectrometer) to determine the $\alpha$-bisabolol content.

\section{Statistical analysis}

Data are presented as mean value of each treatment. The results represent the means \pm standard error with each experiment performed in triplicate. Data were analyzed with one-way ANOVA using SPSS 10.0 for Windows (SPSS Inc., IL, USA), and differences in the treatment means were compared with Duncan's multiple range test at the $P \leq 0.05$ level.

\section{Results}

\section{Effects of ALA treatments on growth}

Compared with the control group, ALA treatments at $1.0,2.0$, and $4.0 \mathrm{mmol} \mathrm{L}^{-1}$ resulted in corresponding $23.0 \%$, $27.0 \%$ and $23.3 \%$ improvement in the dry weight of aboveground, underground, and the total dry weight of $M$. recutita (Fig. 1). Thus, $2.0 \mathrm{mmol}^{-1}$ ALA provided the best results.

\section{Effects of ALA treatments on photosynthesis and chlorophyll content}

1.0, 2.0 and $4.0 \mathrm{mmol}^{-1}$ concentrations of ALA could increase the photosynthetic parameters $(P n, T r, C i$, and $G s)$ of $M$. recutita leaves to different extents (Table 1 ). Treatment using $2.0 \mathrm{mmol}^{-1}$ ALA resulted in the best values for $P n, T r$, and $G s$ at $38 \%, 27 \%$, and $38 \%$ higher than the control group, respectively. $C i$ values at $2.0 \mathrm{mmol}^{-1}$ and 
420

$4.0 \mathrm{mmol}^{-1}$ ALA treatment were significantly higher than those of the control by $30 \%$ and $34 \%$, respectively. These results indicated that photosynthesis was significantly improved by spraying ALA on the leaf of $M$. recutita.
The chlorophyll $a$, chlorophyll $b$, and chlorophyll $(a+$ b) were significantly higher in the leaves of $M$. recutita sprayed with exogenous AIA than in the control (Fig. 2). ALA at $2.0 \mathrm{mmol} \mathrm{L}^{-1}$ showed the best effect, resulting in

Table 1. Gas exchange parameters of $M$. recutita leaves sprayed with different concentrations of ALA

\begin{tabular}{cccc}
\hline $\begin{array}{c}\mathrm{ALA} / \\
\left(\mathrm{mmol} \cdot \mathrm{L}^{-1}\right)\end{array}$ & $\begin{array}{c}P_{n} \\
\left(\mu \mathrm{mol} \mathrm{CO} \cdot \mathrm{m}^{-2} \cdot \mathrm{s}^{-1}\right)\end{array}$ & $\begin{array}{c}T_{r} \\
\left(\mathrm{mmol} \mathrm{H}_{2} \mathrm{O} \cdot \mathrm{m}^{-2} \cdot \mathrm{s}^{-1}\right)\end{array}$ & $\begin{array}{c}C_{i} \\
\left(\mu \mathrm{mol} \mathrm{CO}_{2} \cdot \mathrm{mol}^{-1}\right)\end{array}$ \\
\hline 0 & $9.04+0.25 \mathrm{~b}$ & $8.73+0.18 \mathrm{c}$ & $233.74+10.18 \mathrm{c}$ \\
$(12.25+0.43 \mathrm{a}$ & $8.36+0.26 \mathrm{c}$ & $271.14+13.24 \mathrm{~b}$ & $0.429+0.014 \mathrm{~b}$ \\
2 & $12.46+0.23 \mathrm{a}$ & $11.09+0.35 \mathrm{a}$ & $303.86+18.27 \mathrm{a}$ \\
4 & $12.32+0.57 \mathrm{a}$ & $10.01+0.48 \mathrm{~b}$ & $313.09+11.93 \mathrm{a}$ \\
\hline
\end{tabular}

Each value represents the mean of three replicates of each treatment, and the different normal letters in the same columns mean significant differences at $P<0.05$.
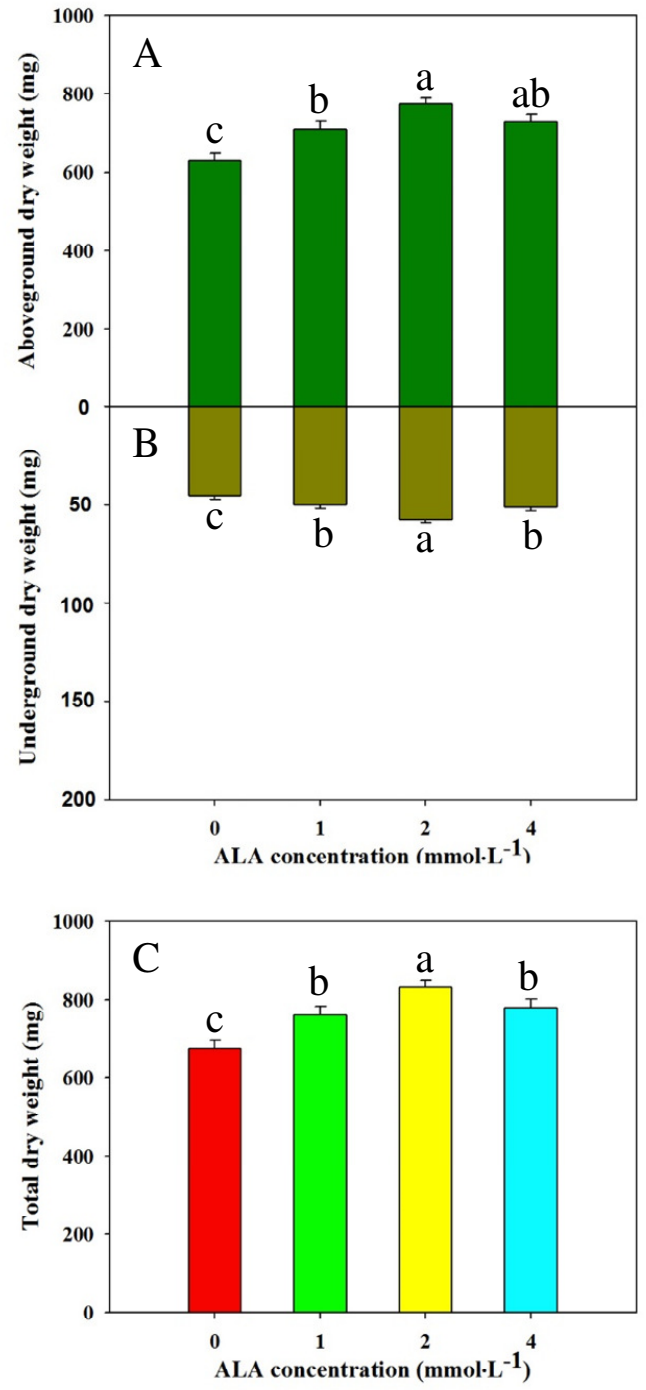

Fig. 1. Dry weight of $M$. recutita treated with different concentrations of 5-aminolevulinic acid (ALA). A, B, and C represent the aboveground dry weight, underground dry weight and total dry weight of $M$. recutita, respectively. Each value represents the mean of three replicates of each treatment, and different normal letters in the same columns indicate significant differences at $P<0.05$

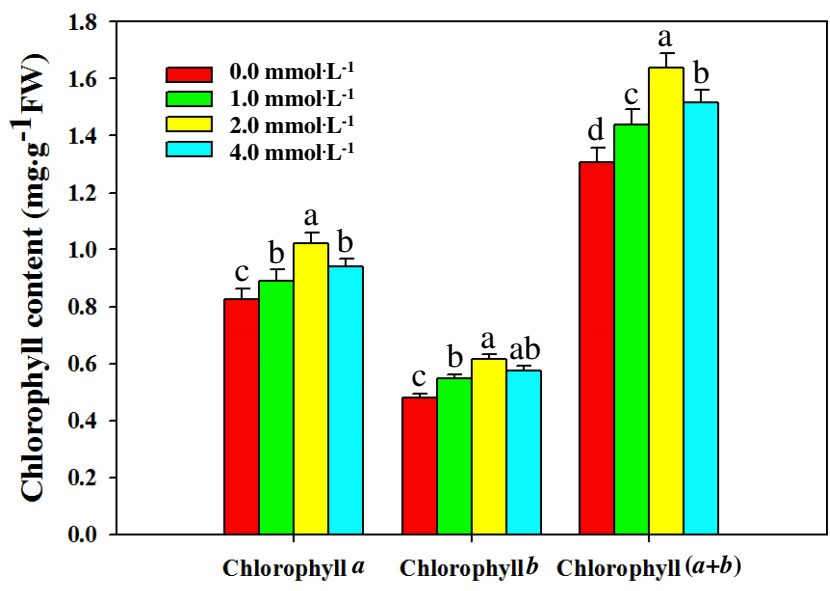

Fig. 2. Chlorophyll content of leaves of $M$. recutita sprayed with different concentrations of ALA. Each value represents the mean of three replicates of each treatment, and different normal letters in the same columns indicate significant differences at $P<0.05$

24\%, 28\%, and 32.1\% higher chlorophyll $a$, chlorophyll $b$ and chlorophyll $(a+b)$ contents, respectively, compared with those of the control group.

\section{Effects of ALA treatments on photosynthetic products}

The contents of photosynthetic products (soluble protein, total amino acids, and soluble sugar) of chamomile under ALA treatments were significantly higher than those of control (Fig. 3). Treatment with $2.0 \mathrm{mmol}^{-1}$ ALA resulted in the highest levels for soluble protein, total amino acids, and soluble sugar, which were 19\%, 39\%, and 33\% higher than that of the control group, respectively. The results indicated that ALA had a significant effect on carbohydrate accumulation.

Effects of ALA treatments on antioxidant enzymatic activities

The activities of SOD, POD, and CAT in $M$. recutita treated with 1.0, 2.0, and $4.0 \mathrm{mmol}^{-1}$ ALA were significantly higher than those in control group (Fig. 4). The 


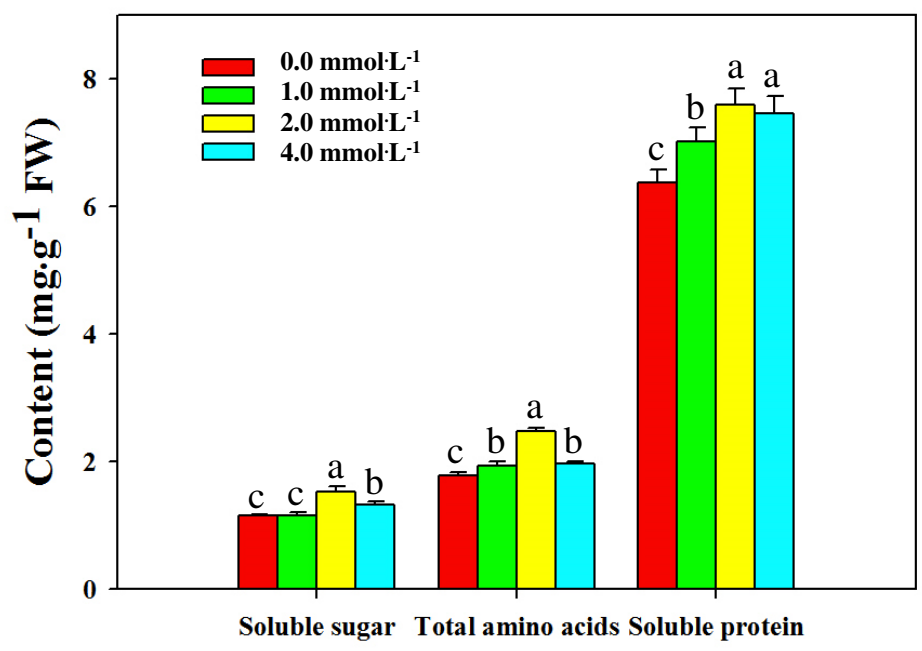

Fig. 3. Effects of ALA treatment on soluble protein, total amino acid, and soluble sugar content of $M$. recutita. Each value represents the mean of three replicates of each treatment, and different normal letters in the same columns indicate significant differences at $P<0.05$
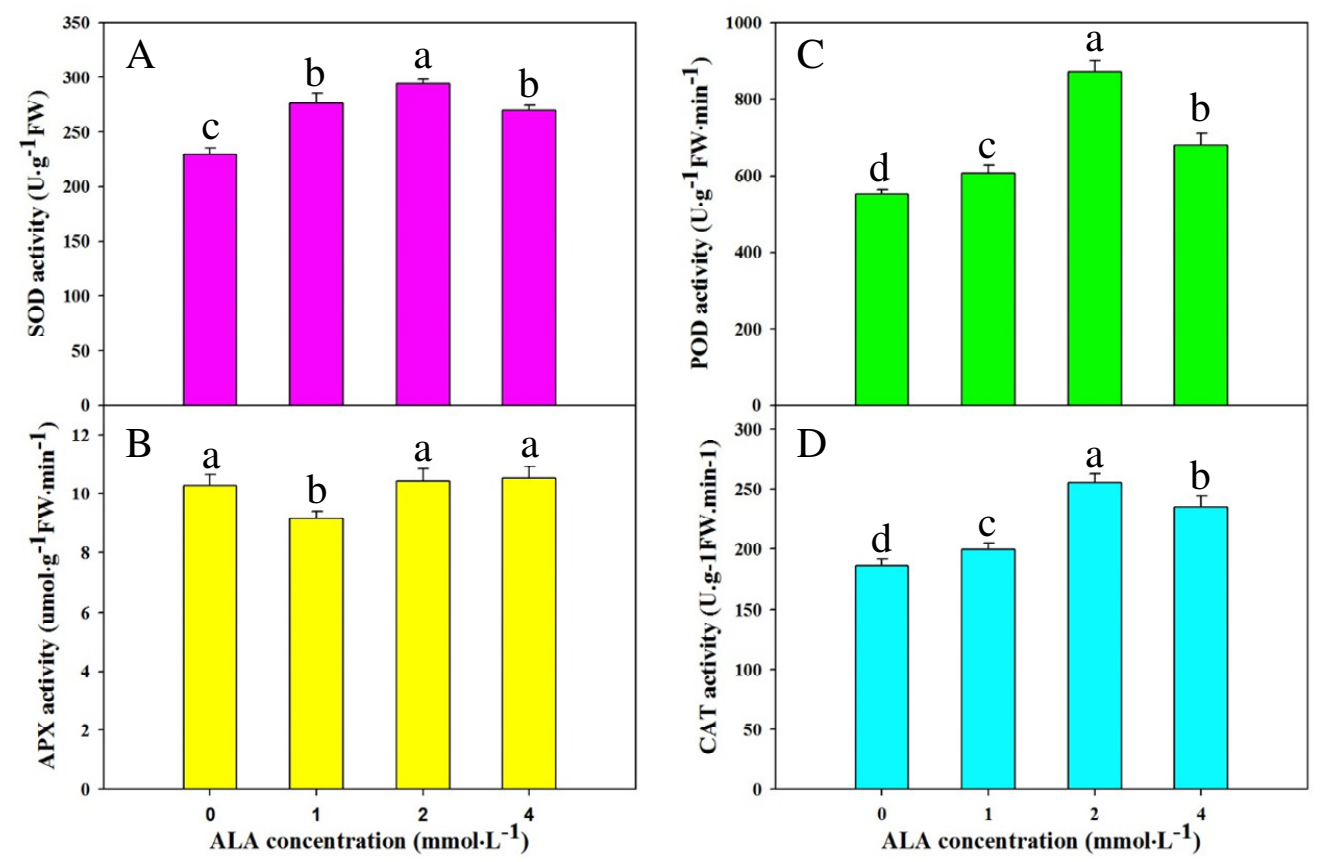

Fig. 4. Activities of superoxide dismutase (SOD), peroxidase (POD), catalase (CAT), and ascorbate peroxidase (APX) of $M$. recutita with ALA treatment. A, B, C and D represent the activity of CAT, SOD, APX and POD enzyme of $M$. recutita under the treatments with different ALA concentrations, respectively. Each value represents the mean of three replicates of each treatment, and the different normal letters in the same columns indicate significant differences at $P<0.05$

highest activity was obtained at $2.0 \mathrm{mmol} \mathrm{L}^{-1}$ ALA treatment with corresponding values $58 \%, 28 \%$, and $37 \%$ higher than in the control. ALA treatment had no effect on APX activity, but APX was significantly lower than that of the control at $2.0 \mathrm{mmol}^{-1} \mathrm{ALA}$.

\section{Effects of ALA Treatment on a-Bisabolol Content}

The content of $\alpha$-bisabolol in $M$. recutita treated with different concentrations of ALA was significantly higher than that of control (Fig. 5). Treatment with $2.0 \mathrm{mmol}^{-1}$ ALA yielded the highest value, which was $21 \%$ higher than the control. This result indicates that ALA can promote the biosynthesis of $\alpha$-bisabolol.

\section{Discussion}

This experiment is the first to verify that exogenous ALA treatment can significantly improve the growth, photosynthesis, antioxidant system, and the $\alpha$-bisabolol of $M$. recutita (Fig. 6). These findings are consistent with those obtained by Akram et al. (2012) in sunflower and Ali et al. (2013) in Brassica napus. Photosynthetic pigments are the 


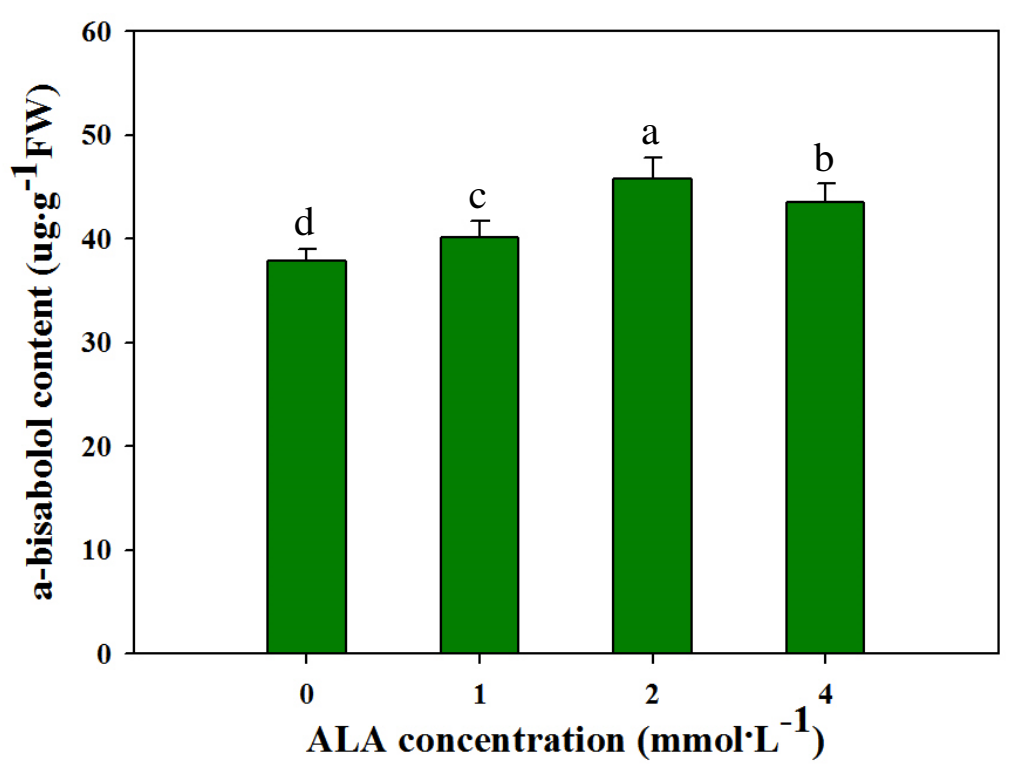

Fig. 5. Effects of ALA treatment on $\alpha$-bisabolol content of $M$. recutita. Each value represents the mean of three replicates of each treatment, and different normal letters in the same columns indicate significant differences at $\mathrm{P}<0.05$

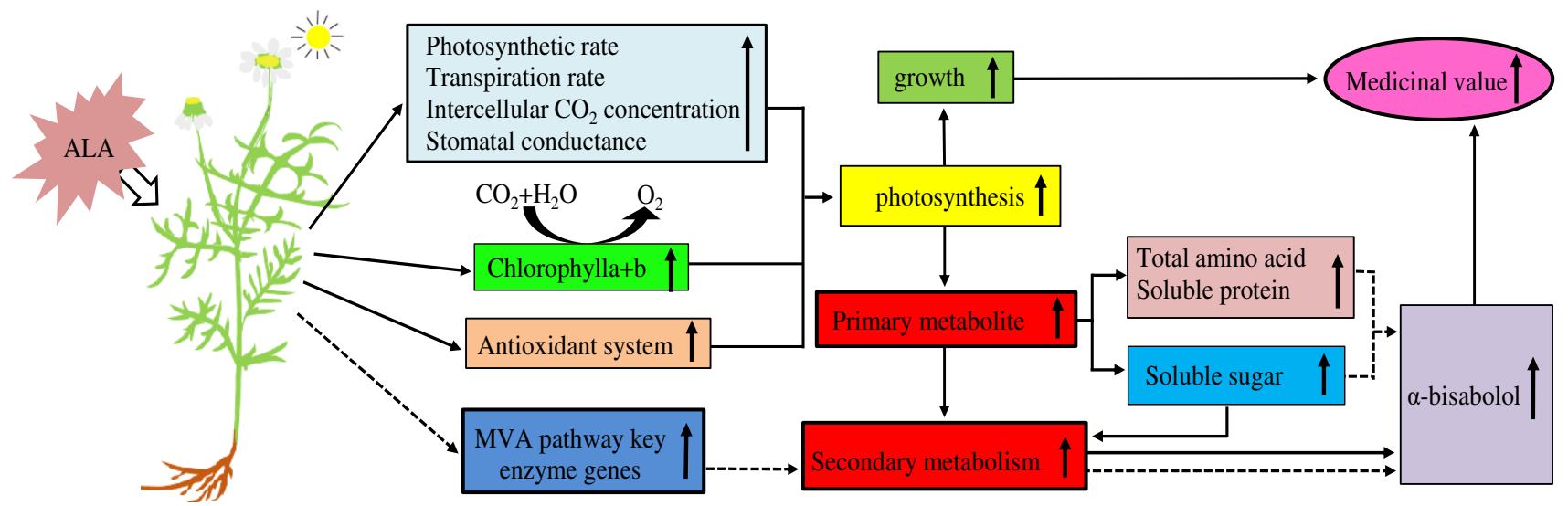

Fig. 6. Simulation diagram of the effects of ALA spraying on $M$. recutita. The dotted line indicates that the result is to be verified in further study. The arrow in the box indicates the increase of content, enzyme activity or metabolic flux

primary factors of plant photosynthesis and are important pigments involved in photosynthesis and in the transfer and transformation of light energy (Castelfranco et al., 1983). Therefore, the photosynthetic pigment in leaves is important, because it reflects the photosynthetic capacity of a plant. The change in environmental factors can alter the photosynthetic pigment content, which in turn varies the photosynthetic performance (Ding et al., 2006). Second, soluble sugar could maintain cell osmotic potential and cell water balance through its osmotic regulation.

Thus, soluble sugar plays a significant role in the physiological effects of photosynthesis. Therefore, the improvement of photosynthesis of $M$. recutita by spraying ALA in this experiment may be due to the increase in photosynthetic pigments and soluble sugar content (Fig. 6). ALA is a precursor material of chlorophyll synthesis and forms protochlorophyllide in the proplastid or chloroplast after a series of enzymatic activities. The protochlorophyllide forms chlorophyll $a$ through the reduction of light, and then chlorophyll $a$ is oxidized to form chlorophyll $b$, preventing the decomposition of chlorophyllase (Wang et al., 2009). The results study show that exogenous ALA treatment could improve the chlorophyll content of $M$. recutita (Fig. 2), and this result is consistent with the results of Hotta et al. (1997b). Thus, the increase in chlorophyll content is one of the reasons for the improved photosynthesis after ALA treatment.

Exogenous ALA treatment significantly increased the content of soluble protein, total amino acids, and soluble sugar (Fig. 3). We inferred that ALA could be a precursor of the chlorophyll biosynthesis, directly involved in photosynthesis of plants, and promote accumulation and transformation of organic matter. Plant photosynthesis is an important source of plant carbon, biomass accumulation, and growth and development. This process can convert inorganic substances into organic matter. Photosynthesis provides the material and energy for plant growth and development (Shao et al., 2009). In addition, ALA 
promotes the accumulation of photosynthetic products. This process may also be related to ALA promotion of plant uptake and utilization of nutrients, because ALA can be combined with trace metal gold and iron and can effectively promote plant growth (Zhang and Zhou, 2000).

This study shows that ALA treatment can improve the activity of antioxidant enzymes in $M$. recutita. This result is consistent with the results of Nishihara et al. (2003) in Spinacia oleracea and Xu et al. (2009) in Ginkgo biloba. SOD is an enzyme that removes superoxide anion from cells, while POD, APX, and CAT are cleared of $\mathrm{H}_{2} \mathrm{O}_{2}$ produced by decomposition of SOD (Leonardis et al., 2000). These enzymes can effectively remove the oxygen free radicals produced during photosynthesis, inhibit membrane lipid peroxidation, and maintain the balance of metabolism of oxygen free radicals, thereby enhancing the resistance of plants (Liangju et al., 1999). Liu et al. (2005) found that promotion of the Pn of winter strawberry leaves by ALA treatment was related to the increase in the leaf SOD and POD activities. Similarly, in this study, ALA treatment was also found to improve the activities of POD, CAT, and SOD in $M$. recutita. POD is an enzyme, with ferroheme as the prothetic group, while ALA is a precursor of the ferroheme biosynthetic. Therefore, the mechanism by which the antioxidant enzyme activity of $M$. recutita cells was increased may be that ALA was converted to ferroheme, and its activity was increased by increasing the prothetic group of the antioxidant enzyme (Huystee et al., 1997). This process thereby reduced the damage of membrane lipid peroxidation, which maintained the integrity of the membrane system and the normal operation of the photosynthetic system and improved photosynthesis (Fig. 6).

Plant primary metabolism through photosynthesis and tricarboxylic acid (TCA) cycle provides energy and a few small molecular compound materials for secondary metabolism. Secondary metabolism is the continuation and development of primary metabolism under specific conditions to avoid the excessive accumulation of certain intermediates or products in the primary metabolic process of toxic effects on the body (Wahid et al., 2007). The secondary metabolites of plants resulted from the interaction between plants during long-term evolution. Many secondary metabolites are important components of traditional Chinese herbal medicine. Among these compounds, alkaloids, flavonoids, saponins, terpenoids, and other compounds, which are indirectly associated with photosynthesis, are the material bases for the efficacy of many traditional Chinese medicines (Su et al., 2005). Plants combine $\mathrm{CO}_{2}$ and water into sugars by photosynthesis, and in different manners produce adenosine triphosphate (ATP), coenzyme (NADH), pyruvate (PA), phosphorenolpyruvic acid (PEP), 4-phosphate-erythrose (E4P), and ribose, which are the indispensable substances in maintaining body life activity. PEP and E4P can further synthesize shikimic acid (CAS). Meanwhile, PA is hydrogenated and decarboxylated to form acetyl-CoA (AcSCoA), enters the TCA cycle, generates a series of organic acids and malonyl-CoA, and obtains a series of amino acids through nitrogen fixation reaction. These processes are the primary metabolic processes. Under certain conditions, several important primary metabolites, such as AcSCoA, malonyl-CoA, CAS, and some amino acids, act as raw materials or substrates, and further different secondary metabolic processes, result in isoprene compounds (e.g. terpenoids), phenolic compounds (e.g. flavonoids) and nitrogen-containing compounds (e.g. alkaloids), and so on (Shao et al., 2008). $\alpha$-Bisabolol is a secondary metabolite of $M$. recutita, a terpenoid compound with multiple therapeutic effects, and synthesized by mevalonate (MVA) pathway. The results of this study show that exogenous ALA spraying on $M$. recutita can increase the $\alpha$-bisabolol content (Fig. 5). Combined with the relationship between secondary metabolism and primary metabolism, we believe that the increase of $\alpha$-bisabolol content of $M$. recutita sprayed ALA may be at the substrate level. PA is a photosynthetic carbon metabolite and also a substrate for the MVA synthesis pathway. Thus, ALA can improve the secondary metabolites of $\alpha$-bisabolol content (Fig. 6). Numerous studies have analyzed the phenotypic and evolutionary patterns of carbon compounds in the distribution of plant secondary metabolites (Heyworth $e t$ al., 1998; Mosaleeyanon et al., 2005). The theory of metabolic overflow indicates that when the carbon element compounds exceed the amount required for plant growth, the excess will be diverted to the synthesis of the plant secondary metabolites (Matsuki et al., 1996). Therefore, the increase in the $\alpha$-bisabolol content in $M$. recutita by ALA treatment may also be attributed to the increase in carbohydrate content, such as soluble sugar, amino acids, and soluble proteins, in the leaves (Fig. 6).

The biosynthetic pathway of chamomile $\alpha$-sweet myrrh has been relatively elucidated. $\alpha$-Bisabolol is synthesized by MVA pathway. This process is mainly based on pyruvic acid derived from TCA as a substrate and forms AcSCoA. Then, $\mathrm{AcSCoA}$ and a series of hydroxylation, acylation, and other enzymatic reaction result in the final synthesis of $\alpha$-bisabolol (Vranová et al., 2012). In recent years, domestic scholars have cloned the several key enzyme gengs in the synthesis of $\alpha$-bisabolol from M. recutita. $\alpha$-Bisabolol content was found to be closely related to the expression level of its key enzyme gene, such as 3-hydroxy-3-methylglutaryl-CoA synthase (HMGS) (Tao et al., 2016a), acetyl-CoA C-acetyltransferase (AACT) (Tao et al., 2016b), and (-)- $\alpha$-bisabolol synthase $(B A S)$ (Son et al., 2014). Exogenous ALA in addition to the physiological level can increase the $\alpha$ bisabolol content, whether it can also regulate the $\alpha$ bisabolol synthesis pathway key enzyme genes expression level to improve the activity of key enzymes, thereby increasing the $\alpha$-bisabolol, still need further study.

\section{Conclusions}

In this experiment, ALA improved the growth, photosynthesis, antioxidant system, and $\alpha$-bisabolol content of $M$. recutita. Treatment with $2.0 \mathrm{mmol} \mathrm{L}^{-1}$ ALA provided the best results. $\alpha$-Bisabolol content was one of the important indices of $M$. recutita medicinal value. Spraying ALA on the leaf can be used as an effective cultivation and control measure to improve the medicinal value of $M$. recutita. ALA is a photosensitive material, that is, it easily decomposes when irradiated. Thus, using ALA is safe to animals, plants, and the environment. The results of this 
424

study provide new and effective cultivation measures to improve the medicinal value and economic value of $M$. recutita.

\section{Acknowledgements}

This work was supported by National Natural Science Foundation of China (Grant no. 31400603).

\section{References}

Akram NA, Ashraf M, Al-Qurainy F (2012). Aminolevulinic acidinduced changes in some key physiological attributes and activities of antioxidant enzymes in sunflower (Helianthus annuus L.) plants under saline regimes. Scientia Horticulturae 142(4):143-148.

Ali B, Wang B, Ali S, Ghani MA, Hayat MT, Yang C, Xu L, Zhou WJ (2013). 5-Aminolevulinic acid ameliorates the growth, photosynthetic gas exchange capacity, and ultrastructural changes under cadmium stress in Brassica napus L. Journal of Plant Growth Regulation 32(3):604-614.

Castelfranco PA, Beale SI (1983). Chlorophyll biosynthesis: recent advances and areas of current interest. Annual Review of Plant Physiology 34(1):241-276.

Ding LN, Jin H, Yin MF, Zhu XJ, Zhao YP, Jiang GB (2006). Effects of salt stress on photosynthetic pigment and gas exchange characteristics of the leaves of Populus seedlings. Acta Botanica Boreali-Occidentalia Sinica 26(12):2523-2527.

Duke SO, Rebeiz CA (1994). Porphyrin biosynthesis as a tool in pest management: an overview. ACS Symposium Series 559(1):1-16.

Farhoudi R (2013). Chemical constituents and antioxidant properties of Matricaria recutita and Chamaemelum nobile essential oil growing wild in the south west of Iran. Journal of Essential Oil Bearing Plants 16(4):531-537.

Heyworth CJ, Iason GR, Temperton V, Jarvis PG, Duncan AJ (1998). The effect of elevated $\mathrm{CO}_{2}$ concentration and nutrient supply on carbon-based plant secondary metabolites in Pinus sylvestris L. Oecologia 115(3):344-350.

Hotta Y, Tanaka T, Luo B, Takeuchi Y, Konnai M (1998). Improvement of cold resistance in rice seedlings by 5 -aminolevulinic acid. Journal of Pesticide Science 23(1):29-33.

Hotta Y, Tanaka T, Takaoka H, Takeuchi Y, Konnai M (1997a). Promotive effects of 5-aminolevulinic acid on the yield of several crops. Plant Growth Regulation 22(2):109-114.

Hotta Y, Tanaka T, Takaoka H, Takeuchi Y, Konnai M (1997b). New physiological effects of 5-aminolevulinic acid in plants: the increase of photosynthesis, chlorophyll content, and plant growth. Bioscience, Biotechnology, and Biochemistry 61(12):2025-2028.

Huystee RBV (1997). Porphyrin and peroxidase synthesis in cultured peanut cells. Canadian Journal of Botany 55(10):1340-1344.

Irmisch S, Krause ST, Kunert G, Gershenzon J, Degenhardt J, Köllner TG (2012). The organ-specific expression of terpene synthase genes contributes to the terpene hydrocarbon composition of chamomile essential oils. BMC Plant Biology 12(1):1-13.

Leonardis SD, Dipierro N, Dipierro S (2000). Purification and characterization of an ascorbate peroxidase from potato tuber mitochondria. Plant Physiology and Biochemistry 38(10):773-779.

Liangju W, Youliang L, Kai M, Guangping L (1999). The changes of antioxidant enzyme activities of fig (Ficus carica l.) cell lines with different salt tolerances. Acta Horticulturae Sinica 26(6):351-355.

Liu D, Pei ZF, Naeem MS, Ming DF, Liu HB, Khan F, Zhou WJ (2011).5-nminolevulinic acid activates antioxidative defence system and seedling growth in Brassica napus L. under water-deficit stress. Journal of Agronomy and Crop Science 197(4):284-295.

Liu W, Kang L, Wang L (2005). Effects on strawberry photosynthesis and relations to antioxidant enzymes of ALA. Acta Botanica Boreali-Occidentalia Sinica 26(1):57-62.

Ma CM, Winsor L, Daneshtalab M (2007). Quantification of spiroether isomers and herniarin of different parts of Matricaria matricarioides and flowers of Chamaemelum nobile. Phytochemical Analysis 18(1):42-49.

Matsuki M (1996). Regulation of plant phenolic synthesis: from biochemistry to ecology and evolution. Australian Journal of Botany 44(6):613-634.

Mosaleeyanon K, Zobayed SMA, Afreen F, Kozai T (2005). Relationships between net photosynthetic rate and secondary metabolite contents in St. John's wort. Plant Science 169(3):523531.

Moustakas M, Sperdouli I, Kouna T, Antonopoulou CI, Therios I (2011). Exogenous proline induces soluble sugar accumulation and alleviates drought stress effects on photosystem II functioning of Arabidopsis thaliana leaves. Plant Growth Regulation 65(2):315325.

Naeem MS, Rasheed M, Liu D, Jin ZL, Ming DF, Yoneyama K, Takeuchi Y, Zhou WJ (2011). 5-Aminolevulinic acid ameliorates salinity-induced metabolic, water-related and biochemical changes in Brassica napus L. Acta Physiologiae Plantarum 33(2):517-528.

Nishihara E, Kondo K, Parvez MM, Takahashi K, Watanabe K, Tanaka K (2003). Role of 5-aminolevulinic acid (ALA) on active oxygen-scavenging system in $\mathrm{NaCl}$-treated spinach (Spinacia oleracea). Journal of Plant Physiology 160(9):1085-1091.

Raal A, Orav A, Püssa T, Valner C, Malmiste B, Arak E (2012). Content of essential oil, terpenoids and polyphenols in commercial chamomile (Chamomilla recutita L. Rauschert) teas from different countries. Food Chemistry 131(2):632-638.

Shao HB, Chu LY, Jaleel CA, Manivannan P, Panneerselvam R, Shao MA (2009). Understanding water deficit stress-induced changes in the basic metabolism of higher plants-biotechnologically and sustainably improving agriculture and the ecoenvironment in arid regions of the globe. Critical Reviews in Biotechnology 29(2):131151.

Shao HB, Chu LY, Shao MA (2008). Calcium as a versatile plant signal transducer under soil water stress. BioEssays 30(7):634-641.

Son YJ, Kwon M, Ro DK, Kim SU (2014). Enantioselective microbial synthesis of the indigenous natural product $(-)-\alpha$-bisabolol by a sesquiterpene synthase from chamomile (Matricaria recutita). Biochemical Journal 463(2):239-248.

Srivastava JK, Shankar E, Gupta S (2010). Chamomile: A herbal 
medicine of the past with bright future. Molecular Medicine Reports 3(6):895-901.

Su WH, Zhang GF, Li XH, Ou XK (2005). Relationship between accumulation of secondary metabolism in medicinal plant and environmental condition. Chinese Traditional and Herbal Drugs 36(9):1415-1418.

Tao T, Chen Q, Meng X, Yan J, Xu F, Chang J (2016a). Molecular cloning, characterization, and functional analysis of a gene encoding 3-hydroxy-3-methylglutaryl-coenzyme A synthase from Matricaria chamomilla. Genes \& Genomics 38(12):179-1187.

Tao T, Liu X, Chang J, Xu F, Yin Y (2016b). Cloning and characterisation of the gene encoding acetyl-coa c-acetyltransferase in Matricaria chamomilla. Journal of Pharmaceutical, Chemical and Biological 4(3):386-393.

Vranová E, Coman D, Gruissem W (2012). Structure and dynamics of the isoprenoid pathway network. Molecular Plant 5(2):318-333.

Wahid A, Gelani S, Ashraf M, Foolad MR (2007). Heat tolerance in plants: an overview. Environmental and Experimental Botany 61(3):199-223.

Wang LJ, Jiang WB, Huang BJ (2004). Promotion of 5-aminolevulinic acid on photosynthesis of melon (Cucumis melo) seedlings under low light and chilling stress conditions. Physiologia Plantarum 121(2):258-264.

Wang W, Zhang X, Yan H, Liang S, Ma J, Yang X (2009). Effects of salt water treatments on gas exchange characteristics, photosynthetic pigment and soluble sugar content of Calligonum spp. at different times. Arid Land Geography 32(4):597-603.
Watanabe K, Tanaka T, Hotta Y, Kuramochi H, Takeuchi Y (2000). Improving salt tolerance of cotton seedlings with 5-aminolevulinic acid. Plant Growth Regulation 32(1):97-101.

Wellburn AR (1994). The spectral determination of chlorophylls a and $\mathrm{b}$, as well as total carotenoids, using various solvents with spectrophotometers of different resolution. Journal of Plant Physiology 144(3):307-313.

Xu F, Chang J, Cheng SY, Zhu J, Li LL, Cheng YWW (2009). Promotive effect of 5-aminolevulinic acid on the antioxidant system in Ginkgo biloba leaves. African Journal of Biotechnology 8(16):3769-3776.

Xu L, Zhang W, Ali B, Islam F, Zhu J, Zhou W (2015). Synergism of herbicide toxicity by 5 -aminolevulinic acid is related to physiological and ultra-structural disorders in crickweed (Malachium aquaticum L). Pesticide Biochemistry and Physiology 125:53-61.

Ye J, Yang X, Chen Q, Xu F, Wang G (2017). Promotive effects of 5aminolevulinic acid on fruit quality and coloration of Prunus persica (L.) Batsch. Scientia Horticulturae 217:266-275.

Zhang WF, Zhang F, Raziuddin R, Gong HJ, Yang ZM, Lu L, Ye QF, Zhou WJ (2008). Effects of 5-aminolevulinic acid on oilseed rape seedling growth under herbicide toxicity stress. Journal of Plant Growth Regulation 27(2):159-169.

Zhang YB, Zhou Y (2000). Plant physiological activity of 5aminolevulinic acid. World Pesticides 3:8-14. 